\title{
A CLASS OF RIESZ-FISCHER SEQUENCES
}

\author{
RUSSELL M. REID
}

(Communicated by Palle E. T. Jorgensen)

\begin{abstract}
It is proved that if $\left\{\lambda_{n}\right\}$ is a sequence of real numbers whose differences are nondecreasing and satisfy $\sum\left(\lambda_{k+1}-\lambda_{k}\right)^{-2}<\infty$, then the set of complex exponentials $\left\{e^{i \lambda_{n} x}\right\}$ is a Riesz-Fischer sequence in $L_{2}[-A, A]$ for every $A>0$, which is to say that for any positive $A$, the equations $\int_{-A}^{A} f(x) e^{i \lambda_{n} x} d x=c_{n}$ admit a solution $f$ in $L_{2}[-A, A]$ for every sequence $\left\{c_{n}\right\}$ in $\ell_{2}$. In particular, if $\lambda_{n}=n^{p}$, then $\left\{e^{i \lambda_{n} x}\right\}$ is a Riesz-Fischer sequence when $p>\frac{1}{2}$.
\end{abstract}

\section{INTRODUCTION}

The study of nonharmonic Fourier series is concerned with completeness, series expansion, and moment properties of sets of complex exponentials $\left\{e^{i \lambda_{n} x}\right\}$ in various function spaces. In this note, attention is restricted to the Hilbert space $L_{2}$ and to the case where the $\lambda_{n}$ are real. Inner products in $\ell_{2}$ are denoted by parentheses; other Hilbert space inner products are denoted by angled brackets $\langle\cdot, \cdot\rangle$, so that in $L_{2}[-A, A],\langle f, g\rangle=\frac{1}{2 A} \int_{-A}^{A} f(x) \bar{g}(x) d x$.

Definition. A sequence $\left\{f_{1}, f_{2}, f_{3}, \ldots\right\}$ of elements of a Hilbert space $H$ is a Riesz-Fischer sequence if the system of equations $\left\langle f, f_{n}\right\rangle=a_{n}$ has at least one solution $f$ in $H$ for every sequence $\left\{a_{n}\right\}$ in $\ell_{2}$, which is to say that its moment space contains $\ell_{2}$.

We take as a starting point the fundamental characterization provided by a theorem of Boas [1], as stated in Young [5, p. 155]:

Theorem. Let $S=\left\{f_{1}, f_{2}, f_{3}, \ldots\right\}$ be a sequence of vectors belonging to a Hilbert space $H$. Then $S$ is a Riesz-Fischer sequence with bound $m>0$ if and only if the inequality

$$
m \sum\left|a_{n}\right|^{2} \leq\left\|\sum a_{n} f_{n}\right\|^{2}
$$

holds for every finite sequence of scalars $\left\{a_{n}\right\}$.

Received by the editors June 15, 1993.

1991 Mathematics Subject Classification. Primary 42A80.

Key words and phrases. Riesz-Fischer sequence, moment problem, nonharmonic Fourier series. 


\section{RESULT}

Theorem. Let $\left\{\lambda_{n}\right\}$ be a sequence of real numbers whose differences are nondecreasing and satisfy

$$
\sum \frac{1}{\left(\lambda_{k+1}-\lambda_{k}\right)^{2}}<\infty
$$

Then the set of complex exponentials $\left\{e^{i \lambda_{n} x}\right\}$ is a Riesz-Fischer sequence in $L_{2}[-A, A]$ for every $A>0$; in other words the equations $\int_{-A}^{A} f(x) e^{i \lambda_{n} x} d x=c_{n}$ admit a solution $f$ in $L_{2}[-A, A]$ for every sequence $\left\{a_{n}\right\}$ in $\ell_{2}$.

Proof. Using $a$ to represent an $\ell_{2}$ sequence $\left\{a_{1}, a_{2}, \ldots\right\}$ and parentheses to indicate the inner product in $\ell_{2}$, inequality (1) is the statement that

$$
\frac{(G a, a)}{(a, a)} \geq m
$$

where the $\ell_{2}$ operator $G$ is the Gram matrix of the set $\left\{f_{1}, f_{2}, f_{3}, \ldots\right\}$, whose entries are $g_{i j}=\left\langle f_{i}, f_{j}\right\rangle$. It is to be shown that the eigenvalues of finite subsections of $G$ are bounded away from zero, which will follow from two observations:

(1) $G v=0$ implies $v=0$, for every $\ell_{2}$ sequence $v$.

(2) $G=I+M$, where $M$ is a compact operator.

The first condition was proved by Paley and Wiener [3], in their Theorem XLII. For if $G v=0$, then $(G v, v)=0$; Paley and Wiener showed that whenever $\lim _{n \rightarrow \infty}\left(\lambda_{n+1}-\lambda_{n}\right)=\infty$, which follows from the hypotheses of the current theorem, then the exponentials are weakly independent over an arbitrarily short interval: $\sum v_{n} e^{i \lambda_{n} x}=0$ a.e. only when all the $v_{n}$ are zero. Alternatively, one could argue that no set of exponentials satisfying the hypotheses of the theorem could be complete (Young [5] shows that a set of exponentials meeting the hypotheses of this theorem has infinite deficiency in $\left.L_{2}[-A, A]\right)$ and invoke L. Schwarz's [4] observation that an incomplete set of exponentials must be (weakly) independent.

To verify condition (2) above, observe that the diagonal entries of $G=I+M$ are unity and the off-diagonal entries are

$$
g_{n m}=\frac{1}{2 A} \int_{-A}^{A} e^{i \lambda_{n} x} e^{-i \lambda_{m} x} d x=\frac{\sin A\left(\lambda_{n}-\lambda_{m}\right)}{A\left(\lambda_{n}-\lambda_{m}\right)} .
$$

$M$ can be shown to be compact by showing that its Schmidt norm is finite. Since $G$ is symmetric, it suffices to show that

$$
\sum_{i=1}^{\infty} \sum_{j=i+1}^{\infty} g_{i j}^{2}<\infty
$$

That sum is easily bounded above,

(6) $\sum_{i=1}^{\infty} \sum_{j=i+1}^{\infty} g_{i j}^{2}<\frac{1}{A^{2}} \sum_{i=1}^{\infty} \sum_{j=i+1}^{\infty} \frac{1}{\left(\lambda_{j}-\lambda_{i}\right)^{2}}<\frac{1}{A^{2}} \sum_{i=1}^{\infty} \sum_{j=i+1}^{\infty} \frac{1}{\left(\lambda_{i+1}-\lambda_{i}\right)^{2}(j-i)^{2}}$,

where $\left(\lambda_{j}-\lambda_{i}\right) \leq\left(\lambda_{i+1}-\lambda_{i}\right)(j-i)$ follows from the assumption that differences are nondecreasing. Letting $k=j+i$ and recalling the sum of $\left\{1 / k^{2}\right\}$, one can 
conclude that

$$
\sum_{i=1}^{\infty} \sum_{j=i+1}^{\infty} g_{i j}^{2}<\frac{\pi^{2}}{6 A^{2}} \sum_{i=1}^{\infty} \frac{1}{\left(\lambda_{i+1}-\lambda_{i}\right)^{2}}<\infty,
$$

establishing the theorem.

\section{REMARKS}

The argument above applies with minor modifications to doubly infinite sequences $n= \pm 1, \pm 2, \pm 3, \ldots$. One special case of interest is $\lambda_{n}=n^{p}$, which satisfies the hypotheses of the theorem as long as $p>\frac{1}{2}$. If $p=\frac{1}{2}$, the proof above fails, and in fact the operator $M$ does not have finite Schmidt norm.

\section{REFERENCES}

1. R. P. Boas, Jr., A general moment problem; Amer. J. Math. 63 (1941), 361-370.

2. N. Levinson, Gap and density theorems, Academic Press, New York, 1980.

3. R. E. A. C. Paley and N. Wiener, Fourier transforms in the complex domain, Amer. Math. Soc., vol. 19, Providence, RI, 1934.

4. L. Schwarz, Approximation d'une fonction quelconque par des sommes d'exponentielles imaginaires, Ann. Fac. Sci. Toulouse 6 (1943), 111-176.

5. R. M. Young, An introduction to nonharmonic fourier series, Amer. Math. Soc. Colloq. Publ., vol. 26, Amer. Math. Soc., Providence, RI, 1939.

Department of Mathematical Sciences, Michigan Technological University, HoughTON, MichigAN 49931

E-mail address: russell@math.mtu.edu 